\title{
Erratum: Time fluctuations in isolated quantum systems of interacting particles [Phys. Rev. E 88, 032913 (2013)]
}

Pablo R. Zangara, Axel D. Dente, E. J. Torres-Herrera, Horacio M. Pastawski, Aníbal Iucci, and Lea F. Santos

(Received 11 October 2013; published 24 October 2013)

DOI: 10.1103/PhysRevE.88.049904

PACS number(s): 05.45.Mt, 05.30.-d, 75.10.Jm, 02.30.Ik, 99.10.Cd

We report a mistake in the computation of the initial state $|\mathrm{PP}\rangle$ for $L=12,14,20$. This produced the spurious oscillations within the decay of fluctuations as a function of $L$, shown in Fig. 6 of our paper. The correct plot is shown here in Fig. 1.

The correct coefficients $\kappa$ for the state $|\mathrm{PP}\rangle$ in the Table $\mathrm{V}$ of our paper are 0.239 for $\widehat{\mathrm{KE}}, 0.206$ for $\hat{C}_{L / 2, L / 2+1}^{z}$, and 0.240 for $\hat{s}_{f}^{z}(\pi)$. The error does not alter any of our observations and conclusions. On the contrary, since the oscillations have been removed, the values of $R^{2}$ for the exponential fittings have consistently improved. Therefore, the correct results for $|\mathrm{PP}\rangle$ presented here reinforce the generality of the exponential decay of the fluctuations with $L$ reported in our paper.

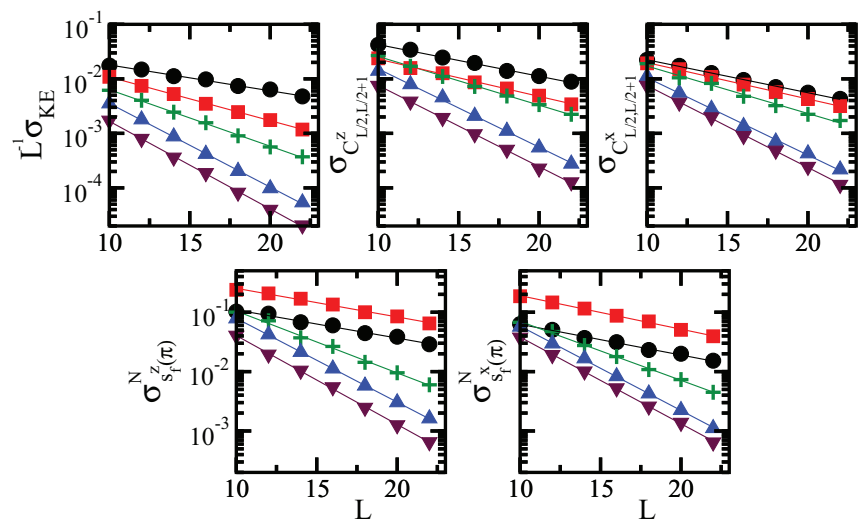

FIG. 1. (Color online) Logarithmic plots of the standard deviation of the time fluctuations for different observables vs $L$ for $|\mathrm{DW}\rangle$ (circles), $|\mathrm{NS}\rangle$ (squares), $|\mathrm{PP}\rangle$ (plus), $\left|\xi_{\mathcal{S}^{z}=0}\right\rangle$ (up triangle), and $\left|\xi_{2}\right\rangle$ (down triangle). The solid lines correspond to logarithmic fits and $\sigma_{O}^{N}=\sigma_{O} / \bar{O}$. All panels: $H_{\Delta=1, \lambda=0}$ and averages performed in the time interval [100,500], except for the domain wall state which used $\left[5 \times 10^{2}, 5 \times 10^{3}\right]$. 